\title{
Measurements of the Isotopic Ratio ${ }^{6} \mathrm{Li} /{ }^{7} \mathrm{Li}$ in Stars with Planets
}

\author{
L. Ghezzi ${ }^{1}$, K. Cunha ${ }^{2,1}$, V. V. Smith², S. Margheim ${ }^{3}$, S. Schuler ${ }^{2}$, F. X. de Araújo ${ }^{1}$ and R. \\ de la Reza ${ }^{1}$
}

\begin{abstract}
High-resolution $(\mathrm{R}=143,000)$, high signal-to-noise $(\mathrm{S} / \mathrm{N}=700-1100)$ Gemini-S bHROS spectra have been analyzed in a search for ${ }^{6} \mathrm{Li}$ in 5 stars which host extrasolar planets. The presence of detectable amounts of ${ }^{6} \mathrm{Li}$ in these mature, solar-type stars is a good monitor of accretion of planetary disk material, or solid bodies themselves, into the outer layers of the parent stars. Detailed profile-fitting of the Li I resonance doublet at $\lambda 6707.8 \AA$ reveals no detectable amounts of ${ }^{6} \mathrm{Li}$ in any star in our sample. The list of stars analyzed includes HD 82943 for which ${ }^{6} \mathrm{Li}$ has been previouly detected at the level of ${ }^{6} \mathrm{Li} /{ }^{7} \mathrm{Li}=$ $0.05 \pm 0.02$. The typical limits in the derived isotopic fraction are ${ }^{6} \mathrm{Li} /{ }^{7} \mathrm{Li} \leq$ 0.00-0.02. These upper limits constrain the amount of accreted material to less than $\sim 0.02$ to 0.5 Jovian masses. The presence of detectable amounts of ${ }^{6} \mathrm{Li}$ would manifest itself as a red asymmetry in the Li I line-profile and the derived upper limits on such asymmetries are discussed in light of three-dimensional hydrodynamic model atmospheres, where convective motions also give rise to slight red asymmetries in line profiles.
\end{abstract}

Subject headings: line: profiles - planetary systems: formation - stars: abundances - stars: atmospheres

\section{INTRODUCTION}

One of the interesting properties of the known stars with planets concerns their metallicity distribution. Several studies (Santos et al. 2000; Gonzalez et al. 2001; Laws et al. 2003; Santos et al. 2005; Fischer \& Valenti 2005) have confirmed the result first shown by Gonzalez

\footnotetext{
${ }^{1}$ Observatório Nacional, Rua General José Cristino, 77, 20921-400, São Cristóvão, Rio de Janeiro, RJ, Brazil; luan@on.br

${ }^{2}$ National Optical Astronomy Observatory, 950 North Cherry Avenue, Tucson, AZ 85719, USA

${ }^{3}$ Gemini Observatory, Casilla 603, La Serena, Chile
} 
(1997): stars with giant planets are systematically metal-rich (by $\sim 0.2$ dex) relative to field FGK dwarfs not known to harbor planets. Two hypotheses have been proposed to explain this excess: primordial enrichment or pollution. The first indicates that the probability of forming giant planets is a steeply rising function of the intrinsic metallicity of the gas and dust cloud which gave birth to the system. This possibility is in agreement with the core-accretion scenario (e.g., Pollack et al. 1996). The higher metal content would raise the surface density of solid material in the disk, leading to a more efficient agglutination of the cores onto which the gas will be accreted. The pollution scenario, on the other hand, indicates that the presence of planets could alter the metallicity of their hosting stars. During the inward migration process of giant planets, solid material from the protoplanetary disk or even inner planetesimals and planets could be accreted into the convective envelope of the hosting star. As this material is depleted in $\mathrm{H}$ and He, the star's metallicity would be enhanced.

Current results (e.g., Fischer \& Valenti 2005) show that the frequency of planets increases significantly for higher metallicities, thus giving strong support for the primordial hypothesis. Evidence for the occurence of pollution is still ambiguous. For instance, Ecuvillon et al. (2006) studied the relation between chemical abundances of several elements and their respective condensation temperatures in stars with and without planets, finding no signifcant differences in the two groups. On the other hand, Pasquini et al. (2007) analyzed the metallicity distributions of planet-hosting dwarfs and giants and found that the latter do not favor metal-rich systems. The authors argue that this result could be a strong indication of pollution, as the metal excess could be erased by the dilution process that takes place during the later stages of stellar evolution.

Another ambiguous result is the possible detection of ${ }^{6} \mathrm{Li}$ in the atmospheres of stars with planets, which is a sensitive test of the pollution hypothesis. Both lithium isotopes are destroyed at relatively low temperatures $\left(T=2 \times 10^{6} \mathrm{~K}\right.$ for ${ }^{6} \mathrm{Li}$ and $T=2.5 \times 10^{6} \mathrm{~K}$ for $\left.{ }^{7} \mathrm{Li}\right)$ in stellar interiors. During the early stages of evolution in solar-type stars (specifically, before entering the main-sequence), these stars are entirely convective and most of the primordial $\mathrm{Li}$ is transported to deeper and hotter layers, where it is rapidly burned. The fraction of lithium destruction is, however, a strong function of the stellar mass. For a given metallicity, there is a mass range in which the ${ }^{6} \mathrm{Li}$ is completely destroyed, while a significant amount of ${ }^{7} \mathrm{Li}$ is preserved. The lower edge of this range falls just above the solar mass (corresponding to main-sequence late-F spectral types) for near solar metallicities. Thus, one should not expect to find any ${ }^{6} \mathrm{Li}$ in the atmospheres of solar-type stars. Any positive detection could indicate an external contamination or pollution process.

Israelian et al. (2001) measured the isotopic ratio ${ }^{6} \mathrm{Li} /{ }^{7} \mathrm{Li}$ in $\mathrm{HD} 82943$ (with two close-in 
giant planets) and found ${ }^{6} \mathrm{Li} /{ }^{7} \mathrm{Li}=0.126 \pm 0.014$. This can be compared to a solar system (meteoritic) ratio of ${ }^{6} \mathrm{Li} /{ }^{7} \mathrm{Li}=0.08$. The positive ${ }^{6} \mathrm{Li}$ detection for this star was interpreted as observational evidence of the pollution process. Reddy et al. (2002) studied ${ }^{6} \mathrm{Li}$ in 8 planet hosting stars (HD 82943 included) and found no significant amount of this isotope in HD 82943, nor in any of the targets analyzed. The difference from the previously published ${ }^{6} \mathrm{Li}$ detection for HD 82943 was attributed to the use of a more complete line list, although it noted the presence of an unidentified absorption in the Li region. Israelian et al. (2003) investigated the nature of the unindentified absorption feature at $6708.025 \AA$, which affects the Li I line, by observing several stars of different effective temperatures. They concluded that a high excitation Si I line first proposed by Müller et al. (1975) is more adequate than the Ti I line used by Reddy et al. (2002). Adopting a revised line list and higher quality spectra, the authors performed a new analysis of HD 82943 and measured ${ }^{6} \mathrm{Li} /{ }^{7} \mathrm{Li}=0.05 \pm$ 0.02. Thus, this most recent result for HD 82943 gave additional support to the pollution scenario.

More recently, Mandell et al. (2004) made a major extension of the previous line lists (especially for the CN contribution) and tested three different possibilities for the unidentified feature at $6708.025 \AA$ (Si I, Ti I and Ti II): for all of the 3 different line lists no ${ }^{6} \mathrm{Li}$ was detected in a sample of three planet-hosting stars. These results are generally consistent with Reddy et al. (2002) and argue against the pollution scenario. Unfortunatelly, HD 82943 was not analyzed by Mandell et al. (2004).

In this paper, the ${ }^{6} \mathrm{Li} /{ }^{7} \mathrm{Li}$ isotopic ratio in $\mathrm{HD} 82943$ is analyzed, as well as in other four planet hosting stars and one star not known to have giant planets. The observations and the data reduction are described in \$2. The analysis procedures including the derivation of atmospheric and broadening parameters and compilation of the line list for the Li I region are presented in $\$ 3$. The abundance results are presented in $\$ 4$ and discussed in $\$ 5$.

\section{OBSERVATIONS AND DATA REDUCTION}

\subsection{Observations}

Spectra of the program stars were obtained at the Gemini-S telescope with the benchmounted High-Resolution Optical Spectrograph (bHROS). Given the brightness of these targets, the "object-only" mode was used for observations; for this mode, a 0.9 " fiber is fed into an image slicer that produces a 'slit', measuring $0.14^{\prime \prime} \mathrm{X} 6.5^{\prime \prime}$ as projected to the camera focal plane. The spectrograph is cross dispersed by a set of fused silica prisms and an image slicer rotation mechanism is used to produce a 'vertical' slit at the observed central 
wavelength on the detector, a single 2048 X 4608 E2V CCD with $13.5 \mu$ m pixels.

The instrument was configured to produce a central wavelength on the detector of 6501 $\AA$. In this configuration, eight incomplete spectral orders were obtained, covering the interval between 5580-7230 $\AA$. The continuous coverage available within a single echelle order varies from $\sim 50-70 \AA$ depending on the order. The detector was used with $1 \mathrm{x} 1$ binning to achieve a resolution close to the spectrograph's nominal resolution of $R=\lambda / \delta \lambda=150,000$ (3 pixel sampling) and minimize the impact of cosmic rays. The actual resolution was measured using 10 ThAr lines in the order containing the Li I feature and this resolution was found to be $\mathrm{R}$ $=143,000 \pm 5000$. The spectra were obtained in a wide range of observing conditions during both classical (2006 May) and queue operations (2006 December and 2007 January). Table1 contains a detailed log of the observations including spectral types, V magnitudes, number of exposures, integration times, and the resulting signal-to-noise ratios (per resolution element). The quoted $\mathrm{S} / \mathrm{N}$ values are based on direct measurements of the rms in sample continuum regions which were selected based on inspection of the Solar Atlas. The measured values of signal-to-noise are compatible with the $\mathrm{S} / \mathrm{N}$ based on Poisson statistics.

In addition to the target spectra, calibration sets of biases, flats, and ThAr arcs were obtained each night. When the observations were done in queue mode, only a single ThAr spectrum was taken each night as only a single object was observed per night. When observations were done in classical mode, ThAr spectra were taken which bracketed the observations. For example, the observations of HD 82943 obtained on May 92006 were bracketed with ThAr spectra and this provides a measure of the spectrograph stability. A direct measurement of the stability can be found when combining multiple spectra via crosscorrelation. The largest drift found was about $3 \mathrm{~m} \AA$ or $1 / 5$ of a pixel. Telluric spectra were also obtained and, as expected, demonstrated no significant atmospheric contamination in the region of the lithium feature.

\subsection{Reduction}

The data were processed using standard echelle reduction practices with IRAF1 packages, following the recipe developed by the bHROS science demonstration team2. The raw spectra were corrected by overscan and bias-image subtraction. The images were then flat-

\footnotetext{
${ }^{1}$ IRAF (Image Reduction and Analysis Facility) is distributed by the National Optical Astronomy Observatories (NOAO), which is operated by the Association of Universities for Research in Astronomy, Inc. (AURA) under cooperative agreement with the National Science Foundation (NSO).

${ }^{2}$ Available at http://www.gemini.edu/sciops/instruments/hros/hrosIndex.html
} 
fielded to remove both pixel-to-pixel variations and small amounts of fringing present in the spectra; since the illumination pattern on the detector is nearly identical for the flat and target spectra, the fringe pattern can be very well removed. Scattered light was then sampled, fit, and subtracted from our images before extraction.

The use of the prism cross disperser combined with the long 'slit' length causes the spectral orders to become tilted away from the central order. The severity of this tilt can potentially result in the loss of resolution in the extraction process. To reduce the impact of the tilts, each of the eight spectral orders was divided into 14 subapertures, for a total of 112 resulting subapertures. Each subaperture was individually extracted with optimal extraction method and blaze corrected with similarly extracted flat-field spectra. The ThAr spectra were also subdivided into the same subapertures and wavelength solutions were derived for each of the 112 subapertures, with a typical rms scatter of $0.001 \AA$. These wavelength solutions were applied to the target subapertures and combined to produce a reduced onedimensional echelle spectrum composed of eight spectral orders. Rejection methods employed in the combination process ensured the removal of cosmic rays from the final spectra. Finally, the spectra were continuum normalized and corrected for radial velocity shifts. More details about the bHROS data reduction process can be found in Schuler et al. (2008). The final processed spectrum for a target star is shown in Figure 1, as an example.

\section{ANALYSIS}

The first step in the analysis is to derive effective temperatures, surface gravities, and microturbulent velocities for the sample stars. In addition, broadening parameters affecting the observed spectral lines also need to be defined. A crucial point in the dertermination of ${ }^{6} \mathrm{Li} /{ }^{7} \mathrm{Li}$ isotopic ratios is the construction of a detailed line list for the region around the $\mathrm{Li} \mathrm{I}$ feature. This section presents a discussion of the analysis method adopted in this study.

\subsection{Line Selection and Derivation of Stellar Parameters}

The bHROS spectra have incomplete wavelength coverage (Section 2.1) which restricts the selection of iron lines to derive stellar parameters and metallicities. A sample of Fe I

and Fe II lines was compiled from the list in Thévenin (1990) and equivalent widths were measured in the solar spectrum (Kurucz et al. 1984) in order to select suitable lines and $g f$-values which produced an abundance scatter of less than or equal to 0.05 dex. The final list of adopted Fe lines, as well as the measured equivalent widths for the target stars and 


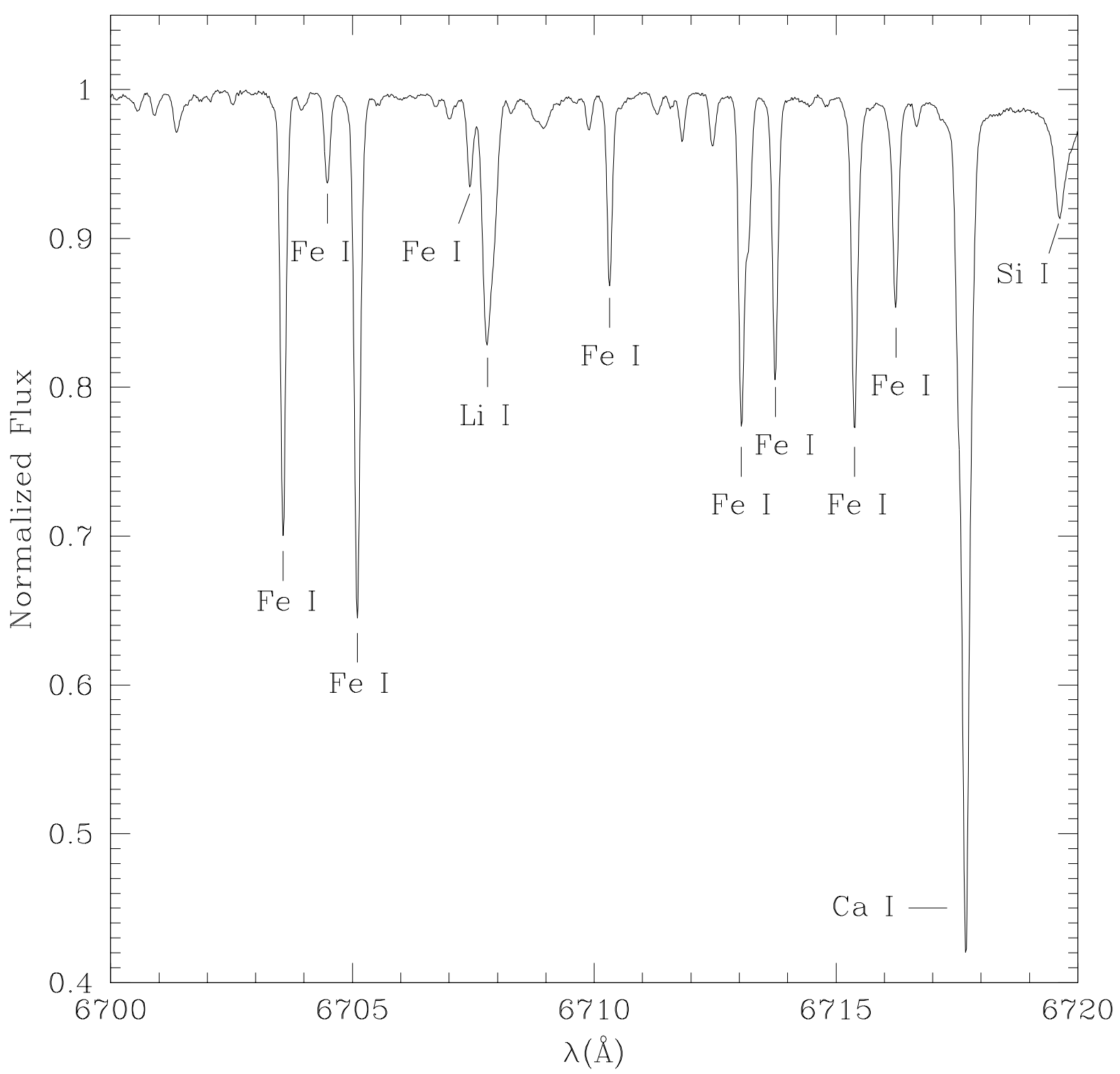

Fig. 1.- The Gemini bHROS spectrum of HD $82943(\mathrm{R} \simeq 143,000$ and $\mathrm{S} / \mathrm{N} \simeq 1130)$, showing part of the spectral order which contains the lithium feature. The main spectral lines in this region are identified. 
the Sun are presented in Table 2. The wavelenghts, lower excitation potentials (LEP), and $g f$-values were taken from the Vienna Atomic Line Databas 3 (VALD-2; Kupka et al. 1999). As a reference note the adopted line list yields a solar abundance $\mathrm{A}(\mathrm{Fe}) 4=7.48 \pm 0.05$ and $\xi=1.24 \mathrm{~km} \mathrm{~s}^{-1}$ using a Kurucz model atmosphere with $T_{\text {eff }}=5777 \mathrm{~K}, \log g=4.44, \xi=$ $2.00 \mathrm{~km} \mathrm{~s}^{-1}$, and $\mathrm{l} / \mathrm{H}_{p}=1.25$.

Stellar parameters for the target stars were derived spectroscopically and followed standard techniques. Effective temperatures were obtained from zero slopes in diagrams of Fe abundance versus excitation potential and surface gravities from ionization equilibrium between Fe I and Fe II species. The microturbulent velocities were varied until the slopes of $\mathrm{A}(\mathrm{Fe} \mathrm{I})$ versus $\log \left(W_{\lambda} / \lambda\right)$ were zero. The abundances were derived in LTE using an updated version of the spectrum synthesis code MOOG5 (Sneden 1973). The model atmospheres adopted in the analysis were interpolated from the ODFNEW grid of ATLAS9 model 6 (Castelli \& Kurucz 2004). Figure 2 shows the iterated result of A(Fe I) versus LEP (top panel) and $\mathrm{A}(\mathrm{Fe} \mathrm{I})$ versus $\log \left(W_{\lambda} / \lambda\right)$ (bottom panel) for the star HD 82943, as an example. The adopted stellar parameters for all target stars are given in Table 3 . Our adopted values for effective temperatures are in good agreement (within $\sim 100 \mathrm{~K}$ ) with the derived values using the photometric correlations presented in Ramírez \& Meléndez (2004). Differences of this amount have no significant effect in the derived isotopic fractions.

The Fe abundance uncertainties can be estimated from the errors in $T_{\text {eff }}, \log g$, $\xi$, line $g f$-values, continuum placement, and equivalent width measurements, among others. We estimate the uncertainties in $T_{\text {eff }}$ to be $\pm 100 \mathrm{~K} ; \log \mathrm{g} \pm 0.2 \mathrm{dex}$; and $\xi \pm 0.2 \mathrm{~km} / \mathrm{s}$. The combined uncertainties in the derived Fe abundances are typical for this type of classical spectroscopic analysis and are estimated to be about \pm 0.1 dex.

The luminosities of the program stars were calculated using apparent V-magnitudes, Hipparcos parallaxes, bolometric corrections from Giradi et al. (2002), and using $\mathrm{M}_{\mathrm{bol}, \odot}$ $=4.77$. No interstellar extinction was included as the most distant star has $\mathrm{d}=65 \mathrm{pc}$. Utilizing these luminosities and the $\mathrm{T}_{\text {eff }}$ values from Table 3, stellar masses were derived by placing the stars in a grid of evolutionary tracks from Girardi et al. (2000) for $[\mathrm{Fe} / \mathrm{H}]=$ 0.00 and 0.20 , with stellar masses covering $\mathrm{M}=0.8$ to $1.4 \mathrm{M}_{\odot}$. Stellar ages were estimated by using the values of effective temperature and luminosity to place the stars in a grid of

\footnotetext{
${ }^{3}$ http://ams.astro.univie.ac.at/ vald/

${ }^{4} \mathrm{~A}(\mathrm{Fe})=\log [\mathrm{N}(\mathrm{Fe}) / \mathrm{N}(\mathrm{H})]+12$

${ }^{5}$ Available at http://verdi.as.utexas.edu/moog.html

${ }^{6}$ http://kurucz.harvard.edu/
} 
isochrones from Girardi et al. (2000) for $[\mathrm{Fe} / \mathrm{H}]=0.00$ and 0.20 , with ages from 0.063 to 7 Gyr. In addition, stellar masses were calculated using the relation between luminosity, surface gravity, and effective temperature:

$$
\log \frac{M}{M_{\odot}}=\log \frac{L}{L_{\odot}}+\log \frac{g}{g_{\odot}}-4 \log \frac{T_{\text {eff }}}{T_{\text {eff }, \odot}}
$$

with $\mathrm{T}_{\text {eff }, \odot}=5777 \mathrm{~K}$ and $\log \mathrm{g}_{\odot}=4.44$. The masses and ages estimated for the targets stars are presented in Table 3 . 
Table 1. Observing log.

\begin{tabular}{ccccccc}
\hline \hline Star & $\begin{array}{c}\text { Spectral } \\
\text { Type }\end{array}$ & $V$ & $\begin{array}{c}\text { Observation } \\
\text { Date }\end{array}$ & $\mathrm{N}$ & $\begin{array}{c}T_{\text {int }} \\
(\mathrm{s})\end{array}$ & $\begin{array}{c}\mathrm{S} / \mathrm{N} \\
(\sim 6708 \AA)\end{array}$ \\
\hline HD 17051 & F9V & 5.40 & 2006 Dec 13 & 1 & 600 & 740 \\
HD 36435 & G9V & 7.01 & 2006 Dec 15 & 2 & 1050 & 560 \\
HD 74156 & G0 & 7.62 & 2007 Jan 30 & 3 & 1320 & 710 \\
HD 82943 & F9V & 6.54 & 2006 May 09,10 & 4 & 600 & 1130 \\
HD 147513 & G1V & 5.37 & 2006 May 10 & 4 & 600 & 1100 \\
HD 217107 & G8IV & 6.16 & 2006 May 10 & 3 & 600 & 750 \\
\hline
\end{tabular}


Table 2. Selected Fe lines and Measured Equivalent Widths.

\begin{tabular}{|c|c|c|c|c|c|c|c|c|c|c|}
\hline \multirow{2}{*}{$\begin{array}{c}\lambda \\
(\AA)\end{array}$} & \multirow[t]{2}{*}{ Ion } & \multirow{2}{*}{$\begin{array}{l}\text { LEP } \\
(\mathrm{eV})\end{array}$} & \multirow{2}{*}{$\begin{array}{r}\log g f \\
(\mathrm{dex})\end{array}$} & \multicolumn{7}{|c|}{$\mathrm{W}_{\lambda}(\mathrm{m} \AA)$} \\
\hline & & & & Sun & HD 17051 & HD 36435 & HD 74156 & HD 82943 & HD 147513 & HD 217107 \\
\hline 5741.848 & $\mathrm{Fe} I$ & 4.26 & -1.730 & 32.9 & 35.4 & 41.9 & 30.8 & 38.8 & 32.1 & 52.7 \\
\hline 5760.345 & $\mathrm{Fe} I$ & 3.64 & -2.490 & 23.8 & 23.5 & 34.9 & 23.4 & 27.6 & 22.7 & 42.0 \\
\hline 5778.453 & $\mathrm{Fe} I$ & 2.59 & -3.430 & 22.7 & 20.9 & 35.6 & 20.7 & 25.0 & 20.8 & 45.5 \\
\hline 5905.672 & $\mathrm{Fe} I$ & 4.65 & -0.863 & 57.9 & 59.9 & 67.9 & 58.3 & 67.3 & 58.1 & 76.7 \\
\hline 5916.247 & $\mathrm{Fe} I$ & 2.45 & -2.832 & 62.2 & 62.9 & 77.6 & 58.5 & 70.0 & 62.7 & 86.2 \\
\hline 5927.789 & $\mathrm{Fe} I$ & 4.65 & -1.090 & 43.1 & $\ldots$ & 52.0 & 42.1 & 57.7 & 42.7 & 67.3 \\
\hline 5930.180 & $\mathrm{Fe} \mathrm{I}$ & 4.65 & -0.028 & 105.7 & 112.1 & 129.4 & 103.2 & 119.4 & 107.7 & 136.1 \\
\hline 6079.009 & $\mathrm{Fe} I$ & 4.65 & -1.120 & 46.2 & 48.3 & 57.6 & 46.0 & 54.7 & 46.8 & 64.3 \\
\hline 6082.711 & $\mathrm{Fe} \mathrm{I}$ & 2.22 & -3.573 & 34.8 & 28.8 & 52.1 & 29.8 & 39.4 & 33.5 & 60.9 \\
\hline 6093.644 & $\mathrm{Fe} \mathrm{I}$ & 4.61 & -1.252 & 31.2 & 33.7 & 40.5 & 34.5 & 43.6 & 31.2 & 54.7 \\
\hline 6094.374 & $\mathrm{Fe} \mathrm{I}$ & 4.65 & -1.661 & 20.2 & 21.7 & 25.3 & 20.2 & 25.1 & 19.5 & 36.9 \\
\hline 6096.665 & $\mathrm{Fe} \mathrm{I}$ & 3.98 & -1.890 & 38.3 & 37.8 & 49.9 & 36.8 & 44.9 & 38.3 & 58.4 \\
\hline 6098.245 & $\mathrm{Fe} \mathrm{I}$ & 4.56 & -1.859 & 16.2 & 18.8 & 23.3 & 16.8 & 21.8 & 16.3 & 33.0 \\
\hline 6265.134 & $\mathrm{Fe} \mathrm{I}$ & 2.18 & -2.550 & 94.0 & 86.0 & 118.6 & 89.4 & 97.6 & $\ldots$ & $\ldots$ \\
\hline 6270.225 & $\mathrm{Fe} I$ & 2.86 & -2.576 & 57.6 & 48.7 & 68.0 & 54.8 & 62.2 & 54.6 & 78.0 \\
\hline 6271.279 & $\mathrm{Fe} \mathrm{I}$ & 3.33 & -2.703 & 23.3 & 23.4 & 34.5 & 21.2 & 27.6 & 22.1 & 42.5 \\
\hline 6297.793 & $\mathrm{Fe} I$ & 2.22 & -2.740 & 81.0 & $\ldots$ & 104.0 & 78.2 & $\ldots$ & 85.1 & 105.7 \\
\hline 6302.494 & $\mathrm{Fe} I$ & 3.69 & -0.973 & 96.1 & 89.8 & $\ldots$ & 91.5 & 103.4 & 101.9 & 123.0 \\
\hline 6315.812 & $\mathrm{Fe} \mathrm{I}$ & 4.08 & -1.710 & 41.7 & 41.9 & 52.3 & 43.0 & 50.4 & 42.4 & 62.6 \\
\hline 6322.686 & $\mathrm{Fe} \mathrm{I}$ & 2.59 & -2.304 & 84.5 & 74.0 & 103.6 & 81.5 & 92.1 & 86.0 & 108.4 \\
\hline 6495.742 & $\mathrm{Fe} \mathrm{I}$ & 4.84 & -0.801 & 48.4 & 45.0 & 58.9 & $\cdots$ & 57.2 & $\cdots$ & 72.4 \\
\hline 6496.467 & $\mathrm{Fe} I$ & 4.80 & -0.348 & 75.6 & 75.6 & 90.8 & 73.4 & 84.8 & 77.8 & 101.6 \\
\hline 6498.939 & $\mathrm{Fe} \mathrm{I}$ & 0.96 & -4.699 & 45.6 & 34.8 & 72.6 & 39.8 & 47.2 & 42.3 & 76.5 \\
\hline 6518.367 & $\mathrm{Fe} \mathrm{I}$ & 2.83 & -2.460 & 66.1 & 62.6 & 80.5 & 62.0 & 72.5 & 62.8 & 87.8 \\
\hline 6699.142 & $\mathrm{Fe} \mathrm{I}$ & 4.59 & -2.101 & 9.1 & 9.2 & 11.9 & 8.2 & 12.0 & 8.0 & 18.0 \\
\hline 6703.567 & $\mathrm{Fe} I$ & 2.76 & -3.141 & 37.4 & 32.2 & 50.9 & 33.5 & 41.6 & 35.2 & 59.4 \\
\hline 6710.320 & $\mathrm{Fe} \mathrm{I}$ & 1.49 & -4.764 & 16.1 & 11.6 & 27.3 & 12.3 & 18.0 & 13.7 & 35.4 \\
\hline 6713.745 & $\mathrm{Fe} I$ & 4.80 & -1.338 & 21.8 & 22.5 & 28.6 & 24.0 & 33.1 & 21.4 & 44.1 \\
\hline 6716.237 & $\mathrm{Fe} I$ & 4.58 & -1.836 & 16.0 & 17.3 & 22.6 & 16.6 & 22.1 & 15.3 & 31.6 \\
\hline 6725.357 & $\mathrm{Fe} \mathrm{I}$ & 4.10 & -2.300 & 17.5 & 16.7 & 23.9 & 17.0 & 22.8 & 17.7 & 34.3 \\
\hline 6726.666 & $\mathrm{Fe} I$ & 4.61 & -1.133 & 46.6 & 47.6 & 59.8 & 45.8 & 56.0 & 46.6 & 66.6 \\
\hline 6732.065 & $\mathrm{Fe} I$ & 4.58 & -2.210 & 7.7 & 8.2 & 9.1 & 7.7 & 10.8 & 7.5 & 16.0 \\
\hline 6733.151 & $\mathrm{Fe} \mathrm{I}$ & 4.64 & -1.437 & 26.4 & 26.8 & 32.7 & 25.9 & 34.5 & 25.5 & 48.6 \\
\hline 6739.522 & $\mathrm{Fe} I$ & 1.56 & -4.950 & 12.0 & 9.8 & 19.4 & 8.0 & 14.0 & 10.6 & 26.9 \\
\hline 6745.101 & $\mathrm{Fe} \mathrm{I}$ & 4.58 & -2.160 & 9.0 & 10.6 & 11.3 & 8.9 & 11.9 & 8.3 & 18.1 \\
\hline 6746.955 & $\mathrm{Fe} \mathrm{I}$ & 2.61 & -4.262 & 4.9 & 4.1 & 9.4 & 4.0 & 5.8 & 3.8 & 11.5 \\
\hline 6971.950 & Fe I & 3.02 & -3.340 & 13.8 & 11.7 & 21.1 & 12.5 & 16.5 & 12.3 & 29.4 \\
\hline 6978.852 & $\mathrm{Fe} \mathrm{I}$ & 2.48 & -2.413 & 86.4 & 84.1 & 113.4 & 79.3 & 92.8 & 88.8 & 108.5 \\
\hline 7179.995 & $\mathrm{Fe} I$ & 1.49 & -4.780 & 20.4 & 14.9 & 33.0 & 14.6 & 22.3 & 19.2 & 42.4 \\
\hline 7189.146 & $\mathrm{Fe} I$ & 3.07 & -2.771 & 40.4 & 35.3 & 53.7 & 37.5 & 45.5 & 38.5 & 62.8 \\
\hline 6084.111 & $\mathrm{Fe} I I$ & 3.20 & -3.780 & 22.0 & 32.2 & 18.5 & 31.7 & 32.8 & 21.6 & 31.0 \\
\hline 6113.322 & $\mathrm{Fe} I I$ & 3.22 & -4.110 & 12.5 & 21.0 & 9.6 & 19.1 & 19.8 & 12.0 & 19.0 \\
\hline 7222.394 & $\mathrm{Fe}$ II & 3.89 & -3.276 & 19.7 & $\cdots$ & $\ldots$ & 29.6 & 29.1 & 19.2 & 27.1 \\
\hline 7224.487 & $\mathrm{Fe} I I$ & 3.89 & -3.226 & 21.3 & 32.4 & 16.3 & 31.8 & 30.5 & 20.0 & $\ldots$ \\
\hline
\end{tabular}




\subsection{Spectrum Synthesis}

Lithium isotopic ratios are measured via spectrum synthesis analysis. The Li I doublet at $\sim 6708 \AA$ is an asymmetric blend of two ${ }^{7} \mathrm{Li}$ lines which consist of 7 hyperfine components, spanning a separation of approximately $0.15 \AA$ between stronger blue and weaker red components. The ${ }^{6} \mathrm{Li}$ lines present the exact same configuration, except with 3 hyperfine levels, with a total separation of approximately $0.15 \AA$ between stronger blue and weaker red components. As the ${ }^{6} \mathrm{Li}$ lines are much weaker, with the stronger one situated almost at the same wavelength as the weaker ${ }^{7} \mathrm{Li}$ component, the former isotope appears as a perturbation, providing an additional small asymmetry to the blend. In addition, the lithium region suffers from the contribution of several blends from metal and molecular CN lines.

\subsubsection{Broadening Parameters}

The stellar projected rotational velocity, $v \sin i$, and macroturbulent velocity, $V_{m}$, are broadening parameters which need to be defined for modelling spectral lines via spectrum synthesis. A good strategy to estimate rotation and macroturbulent velocities is to analyze lines which are isolated, unblended, and with similar strengths to the Li line and then apply the same broadening parameters to the Li I synthesis. A few Fe I lines which fall in the same echelle order as the Li I feature were investigated and the Fe I at $6703.567 \AA$ was identified as a clean line which was used to estimate $v \sin i$ and $V_{m}$ for the studied stars.

A grid of synthetic spectra was computed for combinations of $v \sin i$ and $V_{m}$ varying between 0 and $10 \mathrm{~km} \mathrm{~s}^{-1}$ (with steps of $0.1 \mathrm{~km} \mathrm{~s}^{-1}$ ). Also, we let the iron abundance vary within 0.05 dex of the abundance value which was obtained for that line. Best fits between synthetic and observed line profiles were obtained from a $\chi^{2}$ minimization as follows:

$$
\chi_{r}^{2}=\frac{1}{(d-1)} \sum_{i=1}^{n} \frac{\left(O_{i}-S_{i}\right)^{2}}{\sigma^{2}}
$$

where $O_{i}$ and $S_{i}$ are, respectively, the observed and synthetic normalized fluxes at a wavelength point $i$ across the line profile; $\sigma$ is rms error of the continuum, given by $(\mathrm{S} / \mathrm{N})^{-1}$; $d=n-p$ is the number of degrees of freedom in the fit, where $n$ is the number of points in the observed spectra used in the fit and $p$ is the number of free parameters in the calculation of the synthetic spectra. In this case, $p=5: v \sin i$ and $V_{m} ; r$ (continuum level), w (wavelength) and iron abundance $\mathrm{A}(\mathrm{Fe})$. Small adjustments in the continuum level $(r \leq 0.4 \%)$, to

account for possible errors in the normalization process were allowed. In addition, shifts in the central wavelenghts of the Fe I lines were needed in order to properly match the observed lines. 

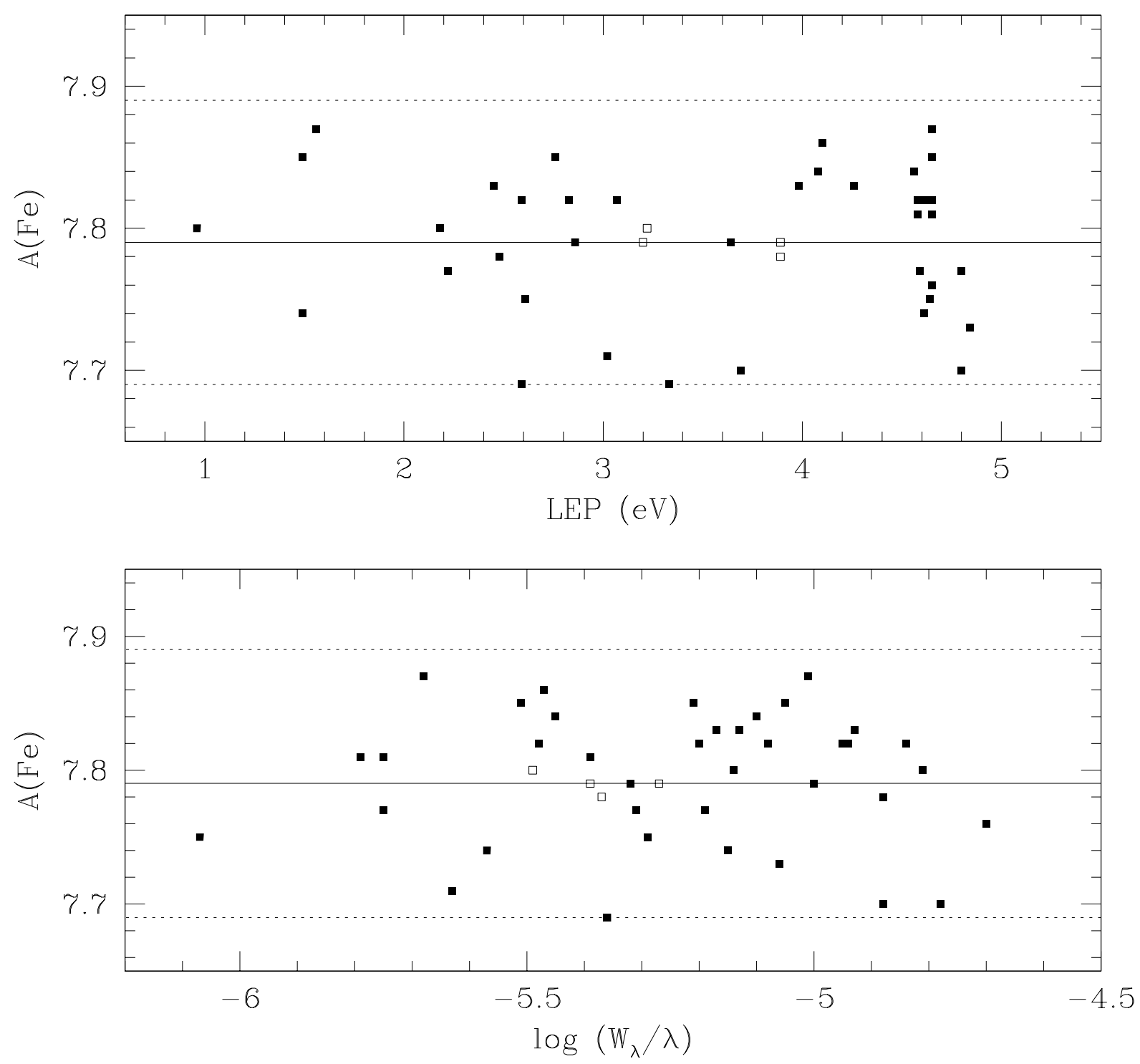

Fig. 2.- Spectroscopic determination of the effective temperature and microturbulent velocity for HD 82943 obtained from zero slopes in the runs of Fe I abundances with excitation potential of the transitions (upper panel) and reduced equivalent widths (lower panel). Fe I (filled squares) and Fe II (open squares) abundances are consistent and the slopes are zero for $\mathrm{A}(\mathrm{Fe})=7.79$. 
Since the resolution of the bHROS spectra is $\mathrm{R} \sim 143,000$, the velocity resolution is just a bit larger than $2 \mathrm{~km} \mathrm{~s}^{-1}$. Thus, at low values of $v \sin i$ and typical macroturblent velocities in stars of the type studied here, the spectra will not be sensitive to changes in low projected rotational velocities found in the program stars. In order to estimate a lower limit to $v \sin i$ that can be detected significantly with the data here, synthetic spectra were computed for the Fe I $6703.567 \AA$ line with a range of values of $v$ sin $i$, from 1.0 to $3.0 \mathrm{~km}$ $\mathrm{s}^{-1}$, a single macroturbulent velocity of $3.0 \mathrm{~km} \mathrm{~s}^{-1}$ and additionally smoothed to the bHROS resolution, using a model with solar parameters. The synthetic spectra were then sampled at the same scale as bHROS, after which noise was added such that $\mathrm{S} / \mathrm{N}=1,000$. These "degraded" synthetic spectra were then subjected to the same analysis as the real spectra, with the result that for $v \sin i$ values of 3.0 and $2.5 \mathrm{~km} \mathrm{~s}^{-1}$ the proper rotational velocities were recovered to within $0.3 \mathrm{~km} \mathrm{~s}^{-1}$ (close to what the estimated uncertainties are for the target stars). For values of 2.0 and $1.5 \mathrm{~km} \mathrm{~s}^{-1}$, the analysis yielded a lower and constant value of $1.3 \mathrm{~km} \mathrm{~s}^{-1}$, indicating that the bHROS spectra are not necessarily sensitive to values of $v \sin i \leq 1.5-2.0 \mathrm{~km} \mathrm{~s}^{-1}$ for typical macroturbulent velocities.

We note, however, that even though the bHROS spectra are not sensitive to projected rotational velocities of less than about $2.0 \mathrm{~km} \mathrm{~s}^{-1}$, it is still found in the real data that small values of $v \sin i$ provide somewhat better fits to the Fe I line profiles than a combination of larger $v \sin i$ and lower macrotubulence. Although these lower values of $v \sin i$ provide better fits, their detection should not be considered significant, with realistic lower limits of $v \sin i=1.5 \mathrm{~km} / \mathrm{s}$.

Figure 3 shows the fit obtained for the star HD 74156. The top panel plot both the observed and best-fit synthetic Fe I line profile as an illustration of the quality of the fits that are obtained in the analysis. The bottom panel illustrates the $\chi^{2}$-minimization techniques used for estimating both projected rotational velocity (left) and macroturbulence (right).

The uncertainties in the overall line broadening caused by macroturbulent and projected rotational velocities can be estimated by varying $v \sin i$ (while keeping $\mathrm{V}_{\mathrm{m}}$ fixed) in an interval of $1.0 \mathrm{~km} \mathrm{~s}^{-1}$ around its best value, with steps of $0.05 \mathrm{~km} \mathrm{~s}^{-1}$. For each value of $v$ sin $i, \Delta \chi_{\mathrm{r}}^{2}$ $=\chi_{\mathrm{r}}^{2}-\chi_{\mathrm{r}, \min }^{2}$ was calculated and the velocity change which produced a $1 \sigma$ change in $\Delta \chi_{\mathrm{r}}^{2}$ was taken as the uncertainty. The uncertainties in $\mathrm{V}_{\mathrm{m}}$ were obtained in a similar manner. The adopted broadening parameters and uncertainties are presented in Table 4 . 
Table 3. Adopted Stellar Parameters and Derived Metallicities.

\begin{tabular}{ccccccc}
\hline \hline \multicolumn{1}{c}{ Star } & $\begin{array}{c}T_{\text {eff }} \\
(\mathrm{K})\end{array}$ & $\begin{array}{c}\log g \\
\left(\mathrm{~cm} \mathrm{~s}^{-2}\right)\end{array}$ & $\begin{array}{c}\xi \\
\left(\mathrm{km} \mathrm{s}^{-1}\right)\end{array}$ & $\begin{array}{c}\mathrm{M} \\
\left(\mathrm{M}_{\odot}\right)\end{array}$ & $\begin{array}{c}\text { Age } \\
(\mathrm{Gyr})\end{array}$ & $\mathrm{A}(\mathrm{Fe})$ \\
\hline HD 17051 & 6197 & 4.49 & 1.24 & 1.2 & 1.0 & 7.73 \\
HD 36435 & 5503 & 4.56 & 1.46 & 1.0 & 1.0 & 7.52 \\
HD 74156 & 6100 & 4.36 & 1.38 & 1.4 & 2.5 & 7.64 \\
HD 82943 & 6055 & 4.56 & 1.33 & 1.2 & 1.5 & 7.79 \\
HD 147513 & 5904 & 4.63 & 1.48 & 1.0 & 2.0 & 7.52 \\
HD 217107 & 5690 & 4.44 & 1.25 & 1.1 & 5.5 & 7.87 \\
\hline
\end{tabular}

Table 4. Elemental abundances and isotopic ratios.

\begin{tabular}{lcccc}
\hline \hline \multicolumn{1}{c}{ Star } & $\begin{array}{c}v \operatorname{sin~} i \\
\left(\mathrm{~km} \mathrm{~s}^{-1}\right)\end{array}$ & $\begin{array}{c}V_{m} \\
\left(\mathrm{~km} \mathrm{~s}^{-1}\right)\end{array}$ & $\mathrm{A}(\mathrm{Li})$ & ${ }^{6} \mathrm{Li} /{ }^{7} \mathrm{Li}$ \\
\hline Sun & 1.70 & 2.80 & 0.96 & 0.00 \\
HD 17051 & $4.90 \pm 0.25$ & $5.25 \pm 0.30$ & $2.48 \pm 0.01$ & $0.03 \pm 0.04$ \\
HD 36435 & $4.25 \pm 0.20$ & $2.95 \pm 0.30$ & $1.60 \pm 0.03$ & $0.06 \pm 0.08$ \\
HD 74156 & $3.10 \pm 0.25$ & $4.85 \pm 0.25$ & $2.59 \pm 0.01$ & $0.00 \pm 0.03$ \\
HD 82943 & $\leq 1.5$ & $3.60 \pm 0.10$ & $2.49 \pm 0.01$ & $0.00 \pm 0.02$ \\
HD 147513 & $\leq 1.5$ & $3.05 \pm 0.10$ & $2.03 \pm 0.01$ & $0.02 \pm 0.03$ \\
HD 217107 & $\leq 1.5$ & $2.85 \pm 0.10$ & $\leq 0.36$ & $0.00 \pm 0.04$ \\
\hline
\end{tabular}



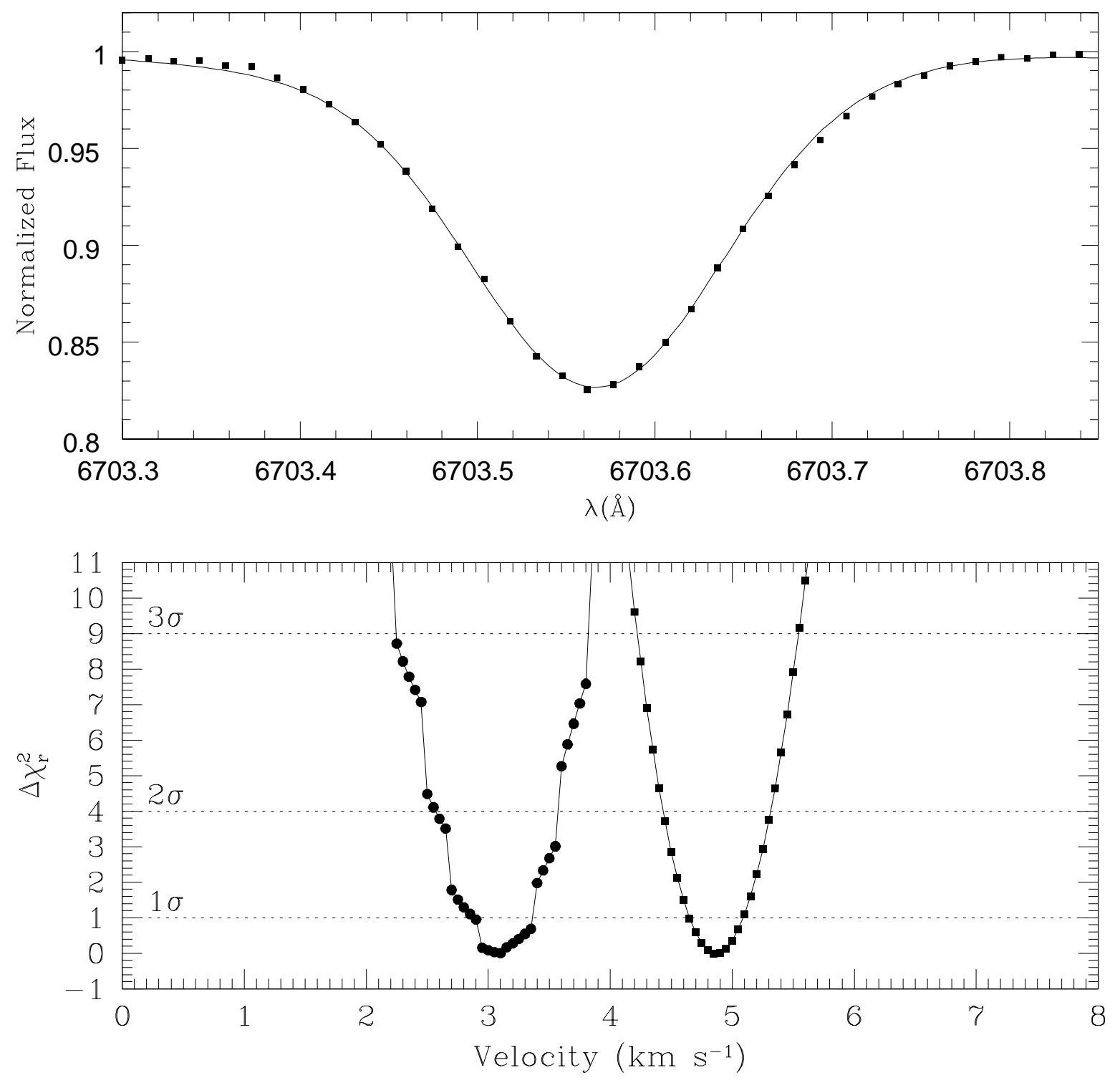

Fig. 3.- Top Panel: Observed (filled squares) and synthetic (solid line) spectra for the selected Fe I line which was used to define the broadening parameters in target star HD 74156. The $\chi_{r}^{2}$-minimization used for estimating $v \sin i$ (filled circles; left) and $\mathrm{V}_{\mathrm{m}}$ (filled squares; right) is shown in the bottom panel. 


\subsubsection{Convection and line asymmetries}

The additional small radial velocity shifts noted above represent convective shifts which are related to the effects of granulation in the stellar atmospheres (see discussion in Allende Prieto et al. 2002). Figure 4 shows the trend of radial velocity shifts applied as a function of the measured equivalent widths for target star HD 82943 and the Sun. The trend of increasingly positive radial velocities as the equivalent widths increase has been noted previously by Allende Prieto et al. (2002), Reddy et al. (2002), and Mandell et al. (2004). All three of these studies measured the Sun and fit straight lines to the trends with slopes and intercepts respectively of: $2.9 \mathrm{~m} \mathrm{~s}^{-1}(\mathrm{~m} \AA)^{-1}$ and $80 \mathrm{~m} \mathrm{~s}^{-1}$ (Allende Prieto et al. 2002); $2.8 \mathrm{~m} \mathrm{~s}^{-1}$ $(\mathrm{m} \AA)^{-1}$ and $120 \mathrm{~m} \mathrm{~s}^{-1}$ (Reddy et al. 2002); $4.2 \mathrm{~m} \mathrm{~s}^{-1}(\mathrm{~m} \AA)^{-1}$ and $30 \mathrm{~m} \mathrm{~s}^{-1}$ (Mandell et al. 2004). Since different Fe lines were used by the different groups, as well as different fitting techniques, the differences between the trends are not surprising and do not lead to significant differences in convective shifts. The results reported here for the Sun are a slope of 2.0 $\mathrm{m} \mathrm{s}^{-1}(\mathrm{~m} \AA)^{-1}$ and an intercept of $-80 \mathrm{~m} \mathrm{~s}^{-1}$.

Solar-type stars are known to display slight red asymmetries in spectral lines due to the convective motions of granules (e.g. Allende Prieto et al. 2002); the flux across the line is dominated by hot, rising granules, while the cooler, falling (i.e., red-shifted) inter-granule regions produce the small red asymmetries. The line-bisector for the Fe I $6703.567 \AA$ line is shown in Figure 15, where it is plotted as flux level versus velocity (in $\mathrm{m} \mathrm{s}^{-1}$ ) instead of wavelength. A detailed discussion of stellar line bisectors can be found in Gray (2005). The filled squares are the observed points in HD 82943, while the open squares were derived from a synthesis of this region using a standard 1D model, which necessarily produces a symmetric line profile. The synthetic profile shows a vertical bisector, indicating an isolated symmetric line (demonstrating that the Fe I $6703.567 \AA$ line is good for determining broadening parameters). The observed line-bisector in HD 82943 deviates to the red due to convective granules; the magnitude of the convective asymmetry is manifested as a $\sim 200$ $\mathrm{m} \mathrm{s}^{-1}$ excursion in the line bisector. The shape and amplitude of this bisector is typical for stars of this type and represents a rather small perturbation of the line profile.

\subsection{The Line List}

The determination of the ${ }^{6} \mathrm{Li} /{ }^{7} \mathrm{Li}$ isotopic ratio rests upon modeling the shape of the Li I line-profile, which depends not only on the isotopic ratios themselves, but also on the stellar broadening mechanisms discussed in the previous section. In addition, the shape of the Li I profile is affected by several weak absorption features that fall within the wavelength region of the various lithium transitions. There are several weak lines of $\mathrm{CN}$ and other metal 


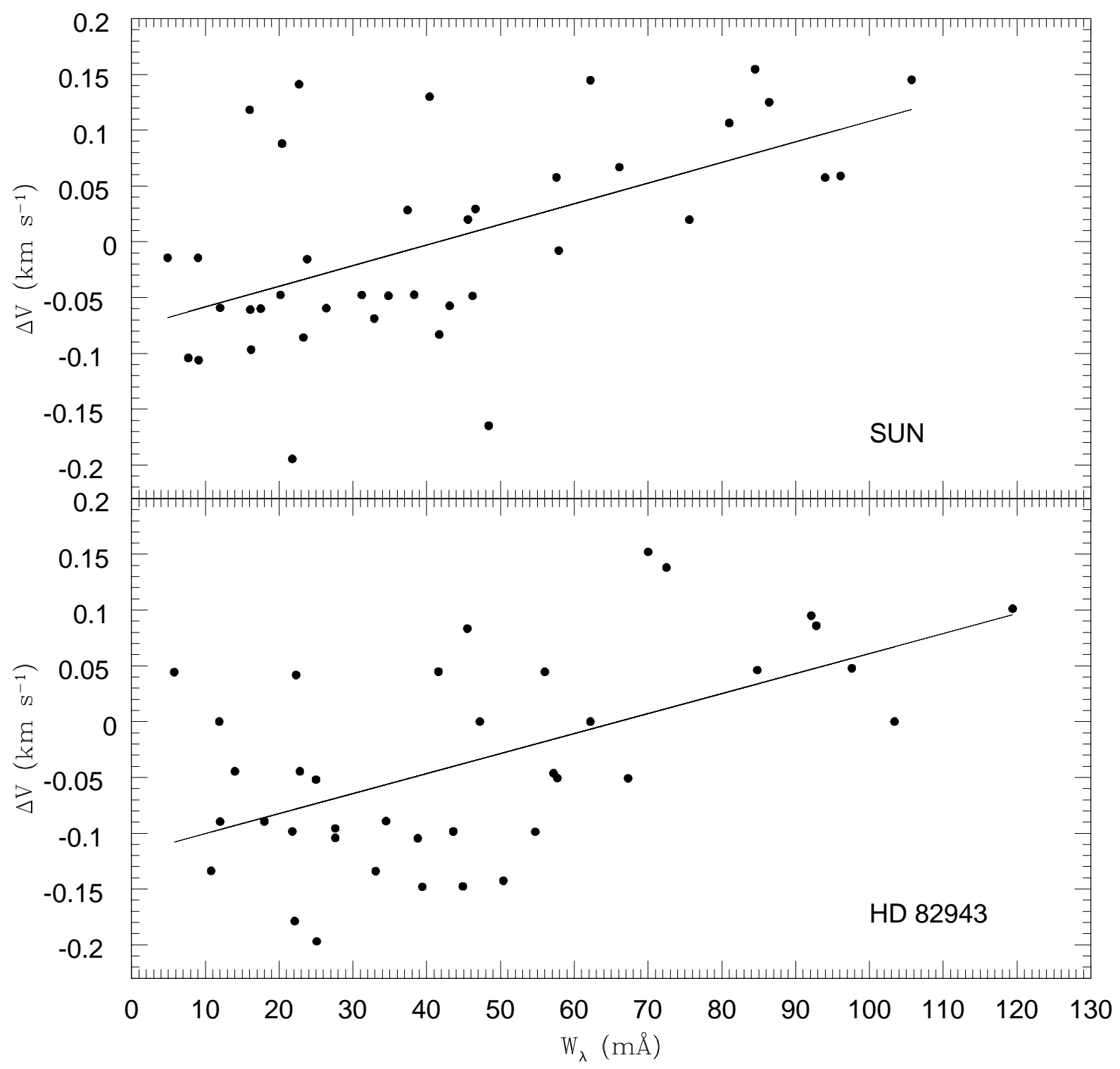

Fig. 4.- Convective shifts measured for Fe I lines in the solar spectrum (top panel) and HD 82943 (bottom panel). The solid lines in each panel represent the least-squares fits to the points. 


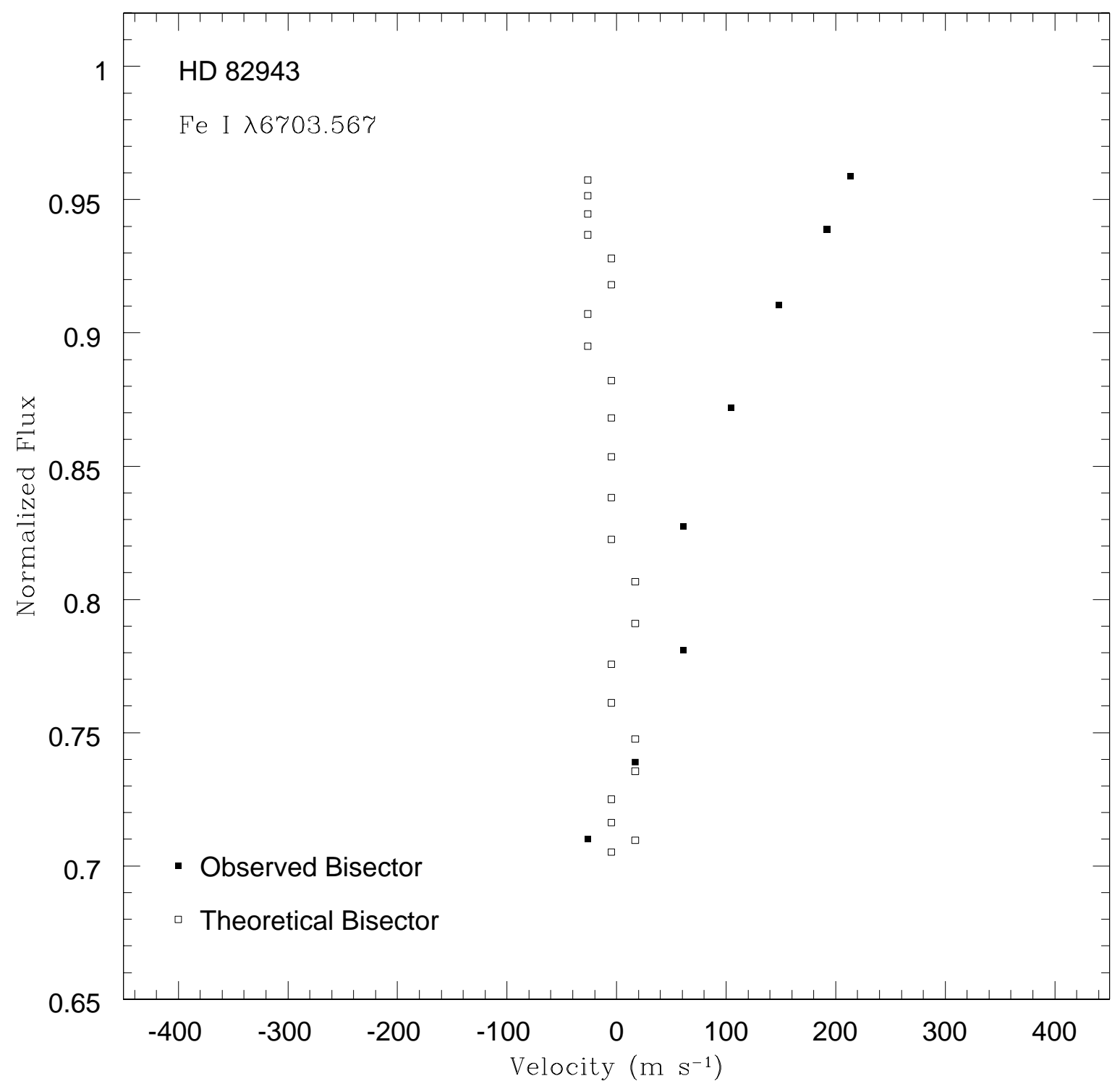

Fig. 5.- Synthetic (open squares) and observed (filled squares) line bisectors measured for Fe I at $6703.567 \AA$. The asymmetry to the red in the observed profile is indicative of photospheric velocity fields. Errors in the bisectors are largest in the central portion of the line. 
absorption lines that will blend with ${ }^{6} \mathrm{Li}$, thus a detailed compilation and careful assessment of line lists is a critical ingredient for the analysis.

As mentioned in the introduction, the search for ${ }^{6} \mathrm{Li}$ in planet hosting stars has been the subject of a few recent studies and line lists for the Li I region are available in the literature. As a starting point in this analysis, we adopted the line list from the most recent and complete study to date by Mandell et al. (2004). The list of lines and atomic data ( $g f$ values and wavelengths) carefully compiled by the authors were adjusted in that study to fit the Kurucz et al. (1984) solar spectrum. In addition, a laboratory carbon arc spectrum was used to adjust the CN lines (in both wavelengths and $g f$-values) in comparison to theoretical line lists. As for the 3 possibilities for the unidentified feature at $6708.025 \AA$ listed by Mandell et al. (2004; Si I, Ti I and Ti II) we adopted the Si I line. Recall that Mandell et al. (2004) found no significant differences in the ${ }^{6} \mathrm{Li} /{ }^{7} \mathrm{Li}$ ratios derived using the 3 different lines. The first step here was to return all atomic data in their line list to those values appearing in the original references. The CN molecular data were kept the same as in Mandell et al. (2004).

This initial line list was checked via comparisons between the solar flux spectrum from Kurucz et al. (1984) and synthetic spectra which are based on the models and synthesis code adopted in this analysis. The solar spectrum was modelled using an ATLAS9 ODFNEW model with $T_{\text {eff }}=5777 \mathrm{~K}, \log g=4.44$, and $\xi=1.24 \mathrm{~km} \mathrm{~s}^{-1}$. The broadening parameters adopted were obtained from fits to the unblended Fe I line at $6703.567 \AA$ : $v$ sin $i=1.70 \mathrm{~km}$ $\mathrm{s}^{-1}, V_{m}=2.80 \mathrm{~km} \mathrm{~s}^{-1}$. The limb darkening coefficient, $\epsilon$, was taken from Van Hamme (1993), however the choice of $\epsilon$ has little effect on the Li I line profile and no measurable impact on the estimation of ${ }^{6} \mathrm{Li}$ isotopic fractions. The comparison of observed solar spectrum and synthesis in the small wavelength interval around the Li I feature was improved by a small continuum adjustment of $0.25 \%$. In addition, small wavelength corrections to the nearby Fe I line $(-0.003 \AA)$ and to Li I $(+0.002 \AA)$ line led to a better fit; these wavelength corrections are those expected due to convective shifts in the solar photosphere (see Section 3.2.2). A small change in the Fe I $g f$-value of +0.015 dex improved the fit to the Fe I profile, while the accurate laboratory $g f$-values for the Li I components were maintained.

Within the scope of the above changes, the fit to the solar spectrum exhibited some small mismatch, especially near 6708.275 $\AA$, where there appeared additional absorption. The absorption in this region was attributed to a $\mathrm{Mg}$ I line by Mandell et al. (2004) and a V I line by Reddy et al. (2002). No references to either of these lines could be found in a literature search, whereas King et al. (1997) attributed the absorption at this wavelength to a Ca I line (present in VALD-2). There were other small differences between the line lists: in the narrow region from 6708.31 to $6708.54 \AA$ the Mandell et al. list contained $4 \mathrm{CN}$ lines while King et al. had 2 (at 6708.375 and $6708.635 \AA$ ). We included the Ca I line plus the two 
adjacent $\mathrm{CN}$ lines; some of the $g f$-values were changed somewhat and wavelengths allowed to shift by $0.001 \AA$ in order to improve the fit to the solar spectrum. The final line list adopted in this study is presented in Table 5. The best-fit to the solar spectrum achieved with this line list was excellent and this is shown in Figure 6. The best-fit was obtained for A(Li) $=0.96$, which is very close to what was found by both King et al. (1997) and Reddy et al. (2002), and within 0.1 dex of the recommended value in Asplund et al. (2005). 
Table 5. The Line List

\begin{tabular}{|c|c|c|c|c|}
\hline $\begin{array}{c}\lambda \\
(\AA)\end{array}$ & Identification & $\begin{array}{l}\text { LEP } \\
(\mathrm{eV})\end{array}$ & $\begin{array}{r}\log g f \\
(\operatorname{dex})\end{array}$ & $\begin{array}{l}\text { Original } \\
\text { Reference }\end{array}$ \\
\hline 6706.548 & $\mathrm{CN} Q_{2}(93)(11,5)$ & 3.130 & -1.359 & M04 \\
\hline 6706.567 & $\mathrm{CN} Q_{2}(80)(8,3)$ & 2.190 & -1.650 & M04 \\
\hline 6706.657 & $\mathrm{CN} R_{12}(22)(7,3)$ & 0.870 & -3.001 & M04 \\
\hline 6706.733 & $\mathrm{CN} Q_{1}(22)(7,3)$ & 0.870 & -1.807 & M04 \\
\hline 6706.844 & $\mathrm{CN} R_{1}(34)(12,7)$ & 1.960 & -2.775 & M04 \\
\hline 6706.863 & $\mathrm{CN} P_{2}(83)(7,2)$ & 2.070 & -1.882 & M04 \\
\hline 6706.880 & Fe II & 5.956 & -4.504 & $\mathrm{~V}$ \\
\hline 6706.980 & Si I & 5.954 & -2.797 & $\mathrm{~V}$ \\
\hline 6707.205 & $\mathrm{CN} Q_{2}(47)(11,6)$ & 1.970 & -1.222 & M04 \\
\hline 6707.282 & $\mathrm{CN} Q_{2}(60)(10,5)$ & 2.040 & -1.333 & M04 \\
\hline 6707.371 & $\mathrm{CN} Q_{1}(85)(12,6)$ & 3.050 & -0.522 & M04 \\
\hline 6707.431 & Fe I & 4.608 & -2.288 & R02 \\
\hline 6707.457 & $\mathrm{CN} P_{12}(13)(7,3)$ & 0.790 & -3.055 & M04 \\
\hline 6707.470 & $\mathrm{CN} Q_{1}(28)(12,7)$ & 1.880 & -1.451 & M04 \\
\hline 6707.473 & Sm II & 0.933 & -1.477 & $\mathrm{~V}$ \\
\hline 6707.518 & V I & 2.743 & -1.995 & V \\
\hline 6707.545 & $\mathrm{CN} Q_{2}(44)(6,2)$ & 0.960 & -1.548 & M04 \\
\hline 6707.595 & $\mathrm{CN} Q_{2}(29)(12,7)$ & 1.890 & -1.851 & M04 \\
\hline 6707.596 & Cr I & 4.208 & -2.767 & V \\
\hline 6707.645 & $\mathrm{CN} P_{21}(44)(6,2)$ & 0.960 & -2.460 & M04 \\
\hline 6707.740 & Ce II & 0.500 & -3.810 & $\mathrm{R} 02$ \\
\hline 6707.752 & Sc I & 4.049 & -2.672 & V \\
\hline 6707.756 & ${ }^{7} \mathrm{Li}$ & 0.000 & -0.428 & H99 \\
\hline 6707.768 & ${ }^{7} \mathrm{Li}$ & 0.000 & -0.206 & H99 \\
\hline 6707.771 & $\mathrm{Ca} \mathrm{I}$ & 5.796 & -4.015 & $\mathrm{R} 02$ \\
\hline 6707.807 & $\mathrm{CN} R_{1}(64)(5,1)$ & 1.210 & -1.853 & M04 \\
\hline 6707.848 & $\mathrm{CN} R_{1}(61)(19,12)$ & 3.600 & -2.417 & M04 \\
\hline 6707.899 & $\mathrm{CN} P_{2}(39)(20,13)$ & 3.360 & -3.110 & M04 \\
\hline 6707.907 & ${ }^{7} \mathrm{Li}$ & 0.000 & -1.509 & H99 \\
\hline
\end{tabular}




\section{LITHIUM ABUNDANCES AND ISOTOPIC RATIOS}

As discussed previously, several blending lines due to metals and CN affect the shape of the Li I feature in metal-rich stars. It is possible to study which of these potentially offending lines have the largest effects on the ${ }^{6} \mathrm{Li}$ absorption via test spectrum syntheses which can isolate each of the nearby lines.

Synthetic spectra were generated to focus on lithium and each of the possible blending species in turn: CN, Si I, Ca I, and V I. In the case of CN, the main impact on ${ }^{6} \mathrm{Li}$ comes from $6707.807 \AA$ which falls slightly blueward $(\sim 0.10 \AA)$ of most of the ${ }^{6} \mathrm{Li}$ absorption and does not affect the derived abundances significantly. The Ca I line at $6708.275 \AA$ falls too far to the red to affect ${ }^{6} \mathrm{Li}$, while V I at $6708.094 \AA$ is too weak to even be detectable. The biggest effect on ${ }^{6} \mathrm{Li}$ absorption comes from the blending line due to Si I at $6708.023 \AA$, where its absorption falls on the red side of possible ${ }^{6} \mathrm{Li}$ absorption. Even here, absorption from ${ }^{6} \mathrm{Li}$ that is about $1 \%$ deep would compete with $\mathrm{Si}$ I (at solar metallicity) and be detectable. These tests indicate that ${ }^{6} \mathrm{Li}$ absorption which is $\sim 1 \%$ deep would be marginally detected, while larger amounts of ${ }^{6} \mathrm{Li}$ that would produce absorption of only a few percent would be detectable.

The important goal is to obtain an overall good fit across the entire Li region from $6707.3 \AA$ to $6708.4 \AA$ and this includes fitting the nearby feature just blueward of Li I which is mainly Fe I with some contribution from CN. Also, a close region nearly free of spectral lines $(6706.40-6706.55 \AA)$ is useful for providing an estimate of the local continuum level, while the wavelengths of Fe I $(6707.431 \AA$ ) and Li I were adjusted slightly to take into account convective shifts (see Figure 44). The abundances of $\mathrm{Ca}$ and $\mathrm{V}$ were allowed to vary within \pm 0.2 dex in order to improve the overall fits to the observed spectra, as well as adjusting $\mathrm{C}$ and $\mathrm{N}$ to fit the blue $\mathrm{CN}$ feature. The final values, or limits, to ${ }^{6} \mathrm{Li}$ were set by a $\chi_{r}^{2}$-minimization of $\mathrm{A}(\mathrm{Li}),{ }^{6} \mathrm{Li} /{ }^{7} \mathrm{Li}$ and $\mathrm{A}(\mathrm{Si})$ simultaneously.

Lithium abundances and isotopic ratios are given in Table 4 for the six target stars. The uncertainties estimated for the $\mathrm{Li}$ abundances and isotopic ratios were derived in a manner similar to that used for the rotational and macroturbulent velocities. The total lithium abundance was varied (but keeping ${ }^{6} \mathrm{Li} /{ }^{7} \mathrm{Li}$ fixed) in steps of $0.001 \mathrm{dex}$, with the $\chi_{r}^{2}$ computed for each value. A change in $\chi_{r}^{2}$ of 1 was taken to define the $\pm 1 \sigma$-value of $\mathrm{A}(\mathrm{Li})$. The uncertainty in the isotopic ratio was determined in an analogous way, with ${ }^{6} \mathrm{Li} /{ }^{7} \mathrm{Li}$ values varied in steps of 0.005 and the total $\mathrm{Li}$ abundance being fixed. The errors in $\mathrm{A}(\mathrm{Li})$ and ${ }^{6} \mathrm{Li} /{ }^{7} \mathrm{Li}$ are also shown in Table 4 .

In order to further test the sensitivity of our method to the isotopic ratios, synthetic spectra were computed for 6 values of the ${ }^{6} \mathrm{Li} /{ }^{7} \mathrm{Li}$ ratio (between 0.00 to 0.05 with a step of 
Table 5-Continued

\begin{tabular}{ccccc}
\hline \hline $\begin{array}{c}\lambda \\
(\AA)\end{array}$ & Identification & $\begin{array}{c}\text { LEP } \\
(\mathrm{eV})\end{array}$ & $\begin{array}{c}\text { log } g f \\
(\mathrm{dex})\end{array}$ & $\begin{array}{c}\text { Original } \\
\text { Reference }\end{array}$ \\
\hline 6707.908 & ${ }^{7} \mathrm{Li}$ & 0.000 & -0.807 & $\mathrm{H} 99$ \\
6707.919 & ${ }^{7} \mathrm{Li}$ & 0.000 & -0.807 & $\mathrm{H} 99$ \\
6707.920 & ${ }^{6} \mathrm{Li}$ & 0.000 & -0.479 & $\mathrm{H} 99$ \\
6707.920 & ${ }^{7} \mathrm{Li}$ & 0.000 & -0.807 & $\mathrm{H} 99$ \\
6707.923 & ${ }^{6} \mathrm{Li}$ & 0.000 & -0.178 & $\mathrm{H} 99$ \\
6707.930 & $\mathrm{CN} Q_{21}(35)(12,7)$ & 1.980 & -1.651 & $\mathrm{M} 04$ \\
6707.964 & $\mathrm{Ti} \mathrm{I}$ & 1.879 & -6.903 & $\mathrm{~V}$ \\
6707.980 & $\mathrm{CN} R_{21}(72)(10,5)$ & 2.390 & -2.027 & $\mathrm{M} 04$ \\
6708.023 & $\mathrm{Si} \mathrm{I}$ & 6.000 & -2.910 & $\mathrm{I} 03$ \\
6708.026 & $\mathrm{CN} R_{2}(35)(12,7)$ & 1.980 & -2.031 & $\mathrm{M} 04$ \\
6708.073 & ${ }^{6} \mathrm{Li}$ & 0.000 & -0.303 & $\mathrm{H} 99$ \\
6708.094 & $\mathrm{~V} \mathrm{I}$ & 1.218 & -3.113 & $\mathrm{~V}$ \\
6708.147 & $\mathrm{CN} P_{2}(42)(11,6)$ & 1.870 & -1.434 & $\mathrm{M} 04$ \\
6708.275 & $\mathrm{Ca} \mathrm{I}$ & 2.710 & -3.377 & $\mathrm{~K} 97$ \\
6708.375 & $\mathrm{CN}$ & 1.979 & -1.097 & $\mathrm{~K} 97$ \\
6708.499 & $\mathrm{CN}$ & 1.868 & -1.423 & $\mathrm{~K} 97$ \\
6708.577 & $\mathrm{Fe} \mathrm{I}$ & 5.446 & -2.728 & $\mathrm{~V}$ \\
6708.635 & $\mathrm{CN} P_{2}(42)(11,6)$ & 1.870 & -1.584 & $\mathrm{M} 04$ \\
\hline
\end{tabular}

References. - (H99) Hobbs et al. (1999); (I03) Israelian et al. (2003); (K97) King et al. (1997); (M04) Mandell et al. (2004); (R02) Reddy et al. (2002); (V) VALD2 (Kupka et al. 1999). 


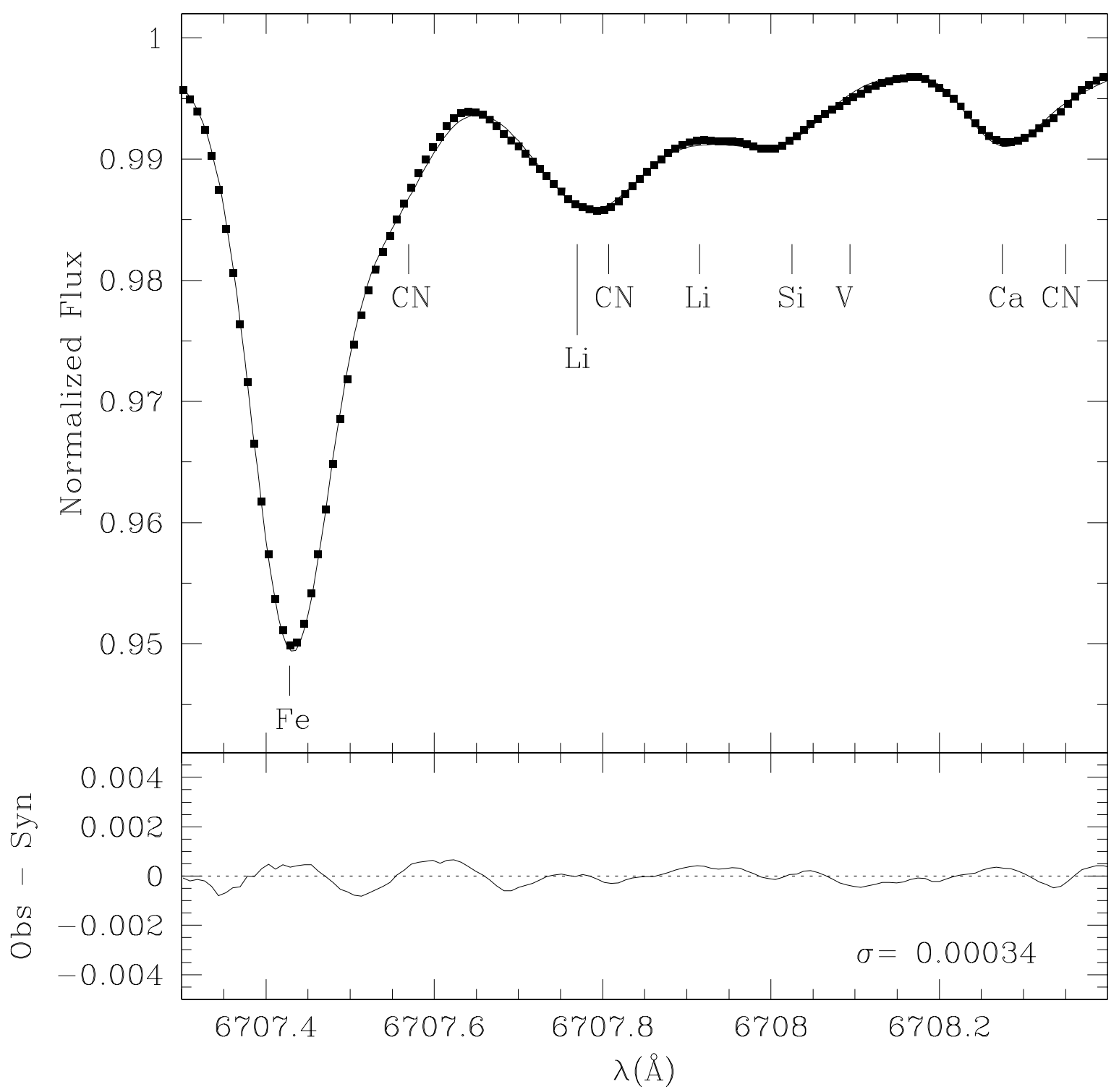

Fig. 6.- Synthetic solar spectrum (solid line) computed with the line list in Table 5. The main lines contributing to the synthesis are identified. The observed solar spectrum (filled squares) is from Kurucz et al. (1984). The bottom panel shows the differences between model and observations; the overall agreement is excellent. 
0.01) adopting the stellar parameters derived for HD 82943. These model spectra were then sampled at the same scale as bHROS, after which noise was added such that $\mathrm{S} / \mathrm{N}=750$ (typical S/N for our observations). These "degraded" synthetic spectra were then subjected to the same analysis as the real spectra and we were able to recover isotopic ratios within \pm 0.01 , demonstrating an analysis sensitivity of 0.01 for ${ }^{6} \mathrm{Li} /{ }^{7} \mathrm{Li}$.

As an additional test, we used the line list in Israelian et al. (2003) in order to analyze the target star HD 82943. The line list adopted in that study is identical to the one used

by Reddy et al. (2002), except for having a Si I line at $6708.025 \AA$ instead of a Ti I line. All the input parameters (model atmospheres, broadening parameters and convective shifts for the Li I and the nearby Fe I line) and the analysis method employed were the same as described above; the only difference being the removal of the $\mathrm{Ca}$ abundance as a free parameter because the feature at $\sim 6708.275 \AA$ is attributed to V I in Israelian et al.'s list. The best fit between model and observations was achieved for $\mathrm{A}(\mathrm{Li})=2.49$ and ${ }^{6} \mathrm{Li} /{ }^{7} \mathrm{Li}=$ 0.01 , which are in excellent agreement with the obtained results using the line list in Table 5 , The derived isotopic ratio for this star, however, is significantly lower than the value derived in Israelian et al. (2003); although the Li abundances in the two studies agree well.

\section{DISCUSSION}

\subsection{Three-Dimensional Model Atmospheres and Their Impact on the One-Dimensional ${ }^{6} \mathrm{Li}$ Results}

As discussed in Section 3.2.2, convective granules result in small red asymmetries in the spectral lines of solar-type stars, such as those analyzed here for ${ }^{6} \mathrm{Li}$. Since the ${ }^{6} \mathrm{Li}$ components of the neutral lithium ground-state doublet fall to the red of the ${ }^{7} \mathrm{Li}$ transitions and because ${ }^{6} \mathrm{Li}$ is expected to be much less abundant than ${ }^{7} \mathrm{Li}$, the presence of ${ }^{6} \mathrm{Li}$ will result in a slight red asymmetry to the combined Li I feature. Since both convection and ${ }^{6} \mathrm{Li}$ can result in a red asymmetric profile, a discussion of the expected convective asymmetries to the Li I feature must be included in order to interpret either detections or upper limits to ${ }^{6} \mathrm{Li}$ fractions.

A more realistic theoretical treatment of the convective motions that cause red-asymmetric line profiles has been included in model stellar atmospheres via the incorporation of radiative hydrodynamical convection, e.g. Stein \& Nordlund (1998) or Asplund et al. (2000). The resulting model atmospheric structures are three-dimensional, time-dependent and contain self-consistent convective flows; such models, often referred to as "3D models", naturally produce absorption lines with small red asymmetries. Recent analyses of the Li I line pro- 

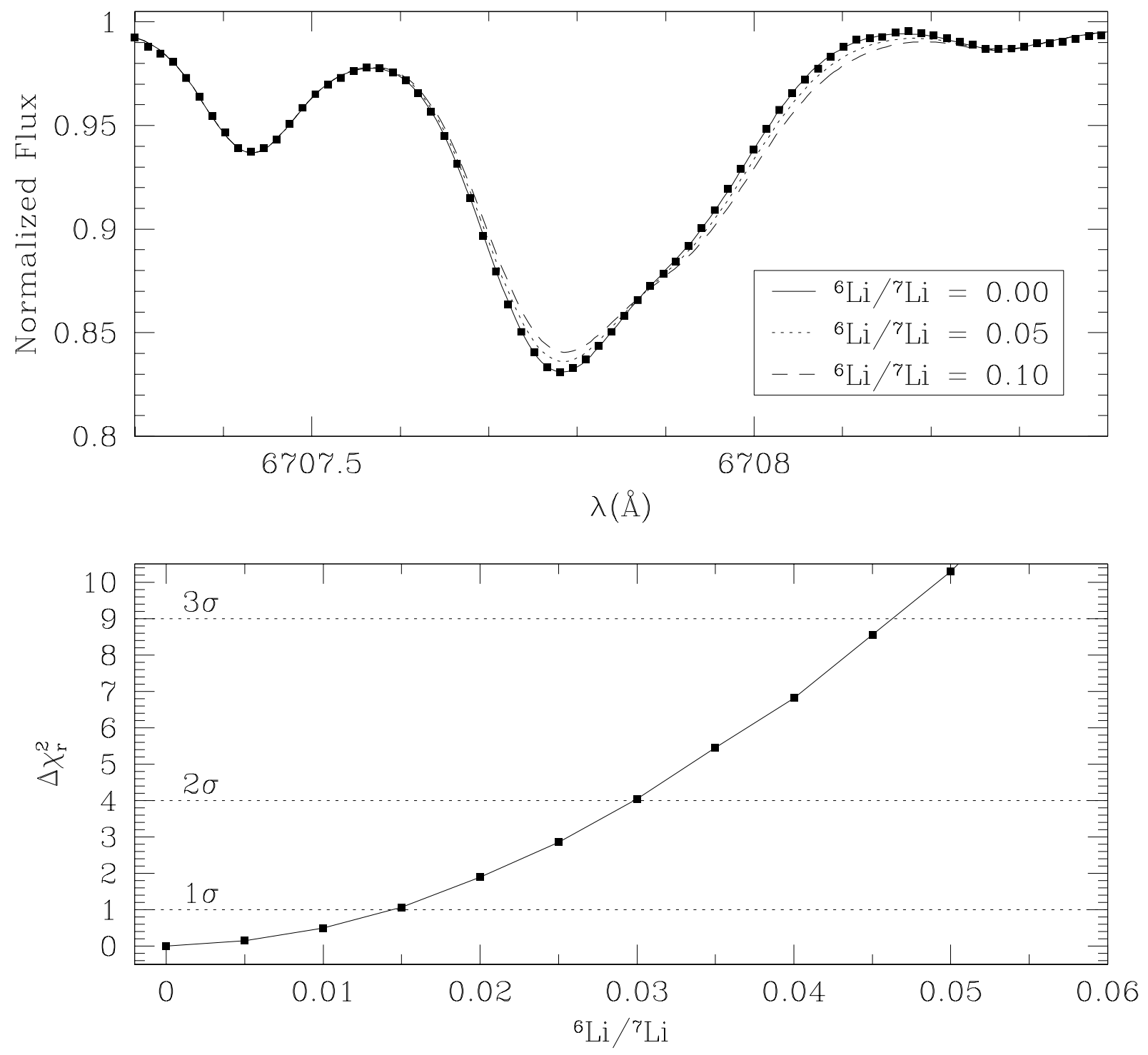

Fig. 7.- Observed and synthetic Li I profiles for HD 82943. The syntheses were calculated for ${ }^{6} \mathrm{Li} /{ }^{7} \mathrm{Li}$ isotopic ratios of 0,5 , and $10 \%$. The Chi-squared minimization of the ${ }^{6} \mathrm{Li} /{ }^{7} \mathrm{Li}$ isotopic ratio is shown in the bottom panel. The best fit is obtained for a synthetic spectrum with zero contribution from ${ }^{6} \mathrm{Li}$. 


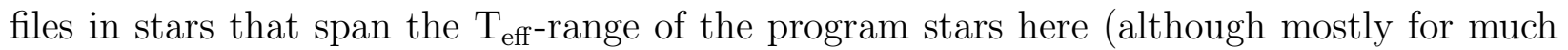
more metal-poor halo dwarfs and subgiants) have included a discussion of effects due to 3D models (Asplund et al. 2006; Cayrel et al. 2007,2008).

Asplund et al. (2006) determined ${ }^{6} \mathrm{Li}$ abundances for a set of 24 metal-poor halo dwarfs and subgiants based on very high quality spectra acquired with the ESO VLT/UVES. The authors found detections of ${ }^{6} \mathrm{Li}$ at the $2 \sigma$ significance level in 9 stars. They also showed that the isotopic ratio ${ }^{6} \mathrm{Li} /{ }^{7} \mathrm{Li}$ is effectively immune to changes in the stellar parameters (at the levels of $\pm 100 \mathrm{~K}, \pm 0.2 \mathrm{dex}, \pm 0.2 \mathrm{dex}$, and $\pm 0.5 \mathrm{~km} / \mathrm{s}$ in $\mathrm{T}_{\text {eff }}, \log \mathrm{g},[\mathrm{Fe} / \mathrm{H}]$, and microturbulent velocity, respectively). Another important result is that the usage of 3D model atmospheres in LTE (when compared to 1D models also in LTE) does not change significantly the derived ${ }^{6} \mathrm{Li}$ fractions from synthesis of the Li I $6707.8 \AA$ line, suggesting that $1 \mathrm{D}$ models are essentially as good as 3D models when employing LTE analyses. Finally, the authors observe that $1 \mathrm{D}{ }^{6} \mathrm{Li} /{ }^{7} \mathrm{Li}$ isotopic ratios are, in general, similar or lower than the ones derived from 3D LTE calculations. The average 3D-1D difference is +0.008 and the largest value is +0.033 . In only 3 cases, are the $3 \mathrm{D}$ values smaller $(-0.010,-0.015$, and $-0.017)$ than the corresponding ones for the $1 \mathrm{D}$ case. These results suggest that the upper limits for the isotopic ratios set here would probably increase if the analysis had been done with 3D. However, the maximum increase would be compatible with the typical errors in the isotopic ratios.

More recently, Cavrel et al. (2007) showed that convection-induced line asymmetries could mimic the presence of ${ }^{6} \mathrm{Li}$, at the few percent level, for the halo turn-off metal-poor star HD 74000. Note that Smith et al. (1998) found ${ }^{6} \mathrm{Li} /{ }^{7} \mathrm{Li}=0.00 \pm 0.02$ for this star via a 1D analysis, similar to the analysis performed in this study. The Cayrel et al. (2007) result was based on a 3D hydrodynamical simulation for a single set of atmospheric parameters, while Cavrel et al. (2008) presented theoretical line asymmetries for a range of atmospheric parameters that cover the values of the stars from Asplund et al. (2006). Note that a 3DNLTE treatment, the most adequate for the Li I analysis according to Asplund et al. (2006), is used by Cayrel $(2007,2008)$. The authors compute an asymmetry of about $2 \%$, which is exactly the mean value of ${ }^{6} \mathrm{Li} /{ }^{7} \mathrm{Li}$ for the stars from Asplund et al. (2006). Also, Cayrel et al. (2008) investigate the dependence of these asymmetries on the stellar parameters $\mathrm{T}_{\text {eff }}, \log \mathrm{g}$, and $[\mathrm{Fe} / \mathrm{H}]$. Based on a small grid of 8 hydrodynamical simulations, they predict an increase in the Li I line asymmetry with metallicity for $[\mathrm{Fe} / \mathrm{H}] \geq-2.0$. Also, they find that hotter stars with lower gravities show larger asymmetries than cooler stars with higher gravities.

Given the tight limits on ${ }^{6} \mathrm{Li}$ fractions derived here via 1D measurements, combined with the Cayrel et al. (2008) predictions that there may be expected line asymmetries of the order of or larger than $\sim 2 \%$ for more metal-rich stars, there is no evidence of detectable ${ }^{6} \mathrm{Li}$ 
in the 6 stars studied here. The average value of ${ }^{6} \mathrm{Li} /{ }^{7} \mathrm{Li}$ for the 6 stars in Table 5 , inversily weighted by their respective uncertainties, is ${ }^{6} \mathrm{Li} /{ }^{7} \mathrm{Li}=0.012$, which is consistent with zero ${ }^{6} \mathrm{Li}$ within the errors. If the non-planet hosting star, HD 36435, is rejected from the average due to its low $\mathrm{S} / \mathrm{N}$ spectrum (Table 1), the weighted average for the 5 stars with planets is even lower: ${ }^{6} \mathrm{Li} /{ }^{7} \mathrm{Li}=0.008$. With limits close to zero, these results also can provide tests on the 3D models and NLTE analyses.

\subsection{Limits on Accreted Mass}

An approximate upper limit to the number of ${ }^{6} \mathrm{Li}$ atoms in the atmospheres of the target stars can be estimated by combining limits to the number of ${ }^{6} \mathrm{Li}$ atoms with the convective zone masses. To carry out this exercise we use the convective zone masses for main sequence stars presented in Pinsonneault et al. (2001) in their Figure 1. This is a simple estimate which assigns a single convective zone mass to the target stars (see table 6) based on their effective temperatures, but will provide a rough upper limit on the number of ${ }^{6} \mathrm{Li}$ atoms present in the outer layers of stellar atmospheres. The ${ }^{6} \mathrm{Li}$ abundance is set by the ${ }^{6} \mathrm{Li} /{ }^{7} \mathrm{Li}$ values in Table 4 ; in those stars where this value is 0.00 , the limit is set to the $1 \sigma$ uncertainty. Table 6 presents the resulting limits to the number of ${ }^{6} \mathrm{Li}$ atoms. In addition, the ${ }^{6} \mathrm{Li}$ abundances are translated to upper limits of accreted mass in Jovian masses. This is an admittedly naive estimate, but does provide a framework in which to limit the amount of accretion that could have occurred on the surfaces of these 5 planet-hosting stars.

Accretion limits of a fraction of a Jovian mass are typical values derived by both Mandell et al. (2004) and Reddy et al. (2002). The tightest accretion limit set here is for the coolest star, HD 217107, due to the increasing strength of the Li I line with decreasing $\mathrm{T}_{\text {eff }}$; this star has a very low total lithium abundance with a corresponding low limit of ${ }^{6} \mathrm{Li}$. The tighter limit on HD 217107 is perhaps also the most interesting from this sample, as

Table 6. Mass Accretion Limits

\begin{tabular}{lccc}
\hline \hline Star & $\begin{array}{c}\log \mathrm{M}_{\mathrm{CZ}} \\
\left(\mathrm{M}_{\odot}\right)\end{array}$ & $\begin{array}{c}\mathrm{N} \\
\left({ }^{6} \mathrm{Li}\right)\end{array}$ & $\begin{array}{c}\mathrm{M}_{\text {acc }} \\
\left(\mathrm{M}_{\text {Jup }}\right)\end{array}$ \\
\hline HD 17051 & -2.44 & $<4.6 \times 10^{43}$ & $<0.33$ \\
HD 74156 & -2.23 & $<1.0 \times 10^{44}$ & $<0.71$ \\
HD 82943 & -2.15 & $<6.2 \times 10^{43}$ & $<0.44$ \\
HD 147513 & -1.90 & $<3.8 \times 10^{43}$ & $<0.27$ \\
HD 217107 & -1.66 & $<2.9 \times 10^{42}$ & $<0.02$ \\
\hline
\end{tabular}


this star is the only one with a very closely orbiting massive planet, with a $\sin i=0.073 \mathrm{AU}$ and $\mathrm{M}_{\text {planet }} \sin i=1.33 \mathrm{M}_{\mathrm{Jup}}$.

While the non-detections of ${ }^{6} \mathrm{Li}$, at levels $1-2 \%$ of total lithium, are secure limits, the interpretation of this lack of ${ }^{6} \mathrm{Li}$ in light of accretion rests on details of stellar evolution. Montalbán \& Rebolo (2002) have presented calculations of the expected evolution of the ${ }^{6} \mathrm{Li}$ abundance as a function of time after ingestion for various stellar model masses and metallicities. Within the standard model framework of stellar evolution, where there is no exchange of material between the convective envelope and the radiative interior, accreted ${ }^{6} \mathrm{Li}$ would survive for gigayears in main-sequence stars with $\mathrm{M} \geq 1.0-1.1 \mathrm{M}_{\odot}$ and having metallicities of $[\mathrm{Fe} / \mathrm{H}]=0.0$ and +0.3 (Montalbán \& Rebolo 2002). These types of stellar masses encompass most of the target stars studied here, thus the limits would suggest that any accretion of planetary material was typically less than a few to several tenths of a Jovian mass.

When stellar evolution with non-standard transport processes, such as microscopic diffusion or turbulent mixing, is used to investigate the fate of ${ }^{6} \mathrm{Li}$ on the surface of solar-type stars, the interpretation becomes more complex, as discussed by Montalbán \& Rebolo (2002). Both diffusion and turbulent mixing act to move material between the convective surface layer and the deeper radiative interior, with the result that accreted ${ }^{6} \mathrm{Li}$ will be removed from the photosphere and destroyed. The efficiency of this destruction is a function of stellar mass, metallicity, and the time at which ${ }^{6} \mathrm{Li}$ is accreted. For warmer effective temperatures, $\mathrm{T}_{\text {eff }} \geq$ $6100 \mathrm{~K}$, the survival of detectable quantities of ${ }^{6} \mathrm{Li}$ can exceed a $\mathrm{Gyr}$, thus if accretion takes place after such stars settle onto the main sequence, there would be measurable amounts of ${ }^{6} \mathrm{Li}$ in some of these stars. Large, sensitive surveys for ${ }^{6} \mathrm{Li}$ remain useful ways to probe and constrain accretion.

\section{CONCLUSIONS}

The main conclusion drawn from the analysis presented here is that no detections of ${ }^{6} \mathrm{Li}$ are found in 5 planet-hosting stars which span the $\mathrm{T}_{\text {eff }}$ range of $5700-6100 \mathrm{~K}$, masses from $\sim 1.1-1.4 \mathrm{M}_{\odot}$, and metallicities from $[\mathrm{Fe} / \mathrm{H}] \sim+0.1-+0.4$. Since the hotter, more massive of the solar-type stars have the least massive convection zones, the stars studied here would be some of the best candidates for detecting signatures of accretion. The combination of high spectral-resolution with high-S/N makes this search one of the most sensitive and the

results here can be combined with previous studies (Reddy et al. 2002; Mandell et al. 2004) to provide strong limits on accretion of ${ }^{6} \mathrm{Li}$. 
We acknowledge the financial support of CNPq. We thank Jeremy King for discussions and for providing the line list for the spectral region between 6700 and $6720 \AA$. KC thanks Martin Asplund for discussions.

Based on observations obtained at the Gemini Observatory, which is operated by the Association of Universities for Research in Astronomy, Inc., under a cooperative agreement with the NSF on behalf of the Gemini partnership: the National Science Foundation (United States), the Science and Technology Facilities Council (United Kingdom), the National Research Council (Canada), CONICYT (Chile), the Australian Research Council (Australia), Ministério da Ciência e Tecnologia (Brazil) and Ministerio de Ciencia, Tecnología e Innovación Productiva (Argentina). Observations were obtained in the following Gemini programs: GS-2006A-C-5 and GS-2006B-Q-47. Research here is supported in-part by NASA GRANT NNH08AJ58I. Support for Simon C. Schuler has been provided by the NOAO Leo Goldberg Fellowship; NOAO is operated by the Association of Universities for Research Astronomy (AURA), Inc., under a cooperative agreement with the National Science Foundation.

Facility: Gemini:South(bHROS)

\section{REFERENCES}

Allende Prieto, C., Asplund, M., García López, R. J., \& Lambert, D. L. 2002, ApJ, 567, 544

Anders, E., Grevesse, N. 1989, Geochim. Cosmochim. Acta, 53, 197

Asplund, M., Grevesse, N., \& Sauval, A. J., in ASP Conf. Ser. 336, Cosmic Abundances as Records of Stellar Evolution and Nucleosynthesis, ed. F. N. Bash \& T. G. Barnes (San Francisco: ASP), 25

Asplund, M., Lambert, D. L., Nissen, P. E., Primas, F., \& Smith, V. V. 2006, ApJ, 644, 229

Asplund, M., Nordlund, A.., Trampedach, R., Allende Prieto, C., \& Stein, R. F. 2000, A\&A, 359,729

Castelli, F., \& Kurucz, R. L. 2004, Proceedings of the IAU Symp. No 210; IAU Symp. No 210, Modelling of Stellar Atmospheres, eds. N. Piskunov et al. 2003, poster A20 (astro-ph/0405087)

Cayrel, R., Steffen, M., Bonifacio, P., Ludwig, H.-G., \& Caffau, E. 2008, arXiv:0810.4290 [astro-ph] 
Cayrel, R., Steffen, M., Chand, H., Bonifacio, P., Spite, M., Spite, F., Petitjean, P., Ludwig, H.-G., \& Caffau, E. 2007, A\&A, 473, L37

Ecuvillon, A., Israelian, G., Santos, N. C., Mayor, M., \& Gilli, G. 2006, A\&A, 449, 809

Fischer, D. A., \& Valenti, J. 2005, ApJ, 622, 1102

Gonzalez, G. 1997, MNRAS, 285, 403

Gonzalez, G. 2006, PASP, 118, 1494

Gonzalez, G., Laws, C., Tyagi, S., \& Reddy, B. E. 2001, AJ, 121, 432

Gray, D. F. 2005, The Observation and Analysis of Stellar Photospheres (3rd ed.; Cambridge: Cambridge Univ. Press)

Hinkle, K., Wallace, L., Valenti, J., \& Harmer, D., ed. 2000, Visible and Near Infrared Atlas of the Arcturus Spectrum 3727-9300 A (San Francisco: ASP)

Hobbs, L. M., Thorburn, J. A., \& Rebull, L. M. 1999, ApJ, 523, 797

Israelian, G., Santos, N. C., Mayor, M., \& Rebolo, R. 2001, Nature, 411, 163

Israelian, G., Santos, N. C., Mayor, M., \& Rebolo, R. 2003, A\&A, 405, 753

Israelian, G., Santos, N. C., Mayor, M., \& Rebolo, R. 2004, A\&A, 414, 601

King, J. R., Deliyannis, C. P., Hiltgen, D. D., Stephens, A., Cunha, K., \& Boesgaard, A. M. 1997, AJ, 113, 1871

Kurucz, R. L., Furelind, I., Brault, J., \& Testerman, L. 1984, Solar Flux Atlas from 296 to 1300 nm (Cambridge: Harvard Univ. Press)

Kupka, F., Piskunov, N., Ryabchikova, T A., Stempels, H. C., \& Weiss, W. W. 1999, A\&AS, 138,119

Laws, C., Gonzalez, G., Walker, K. M., Tyagi, S., Dodsworth, J., Snider, K., \& Suntzeff, N. B. 2003, AJ, 125, 2664

Mandell, A. M., Ge, J., \& Murray, N. 2004, AJ, 127, 1147

Müller, E. A., Peytremann, E., \& de la Reza, R. 1975, Sol. Phys., 41, 53

Montalbán, J., \& Rebolo, R. 2002, A\&A, 386, 1039 
Pasquini, L., Döllinger, M. P., Weiss, A., Girardi, L., Chavero, C., Hatzes, A. P., da Silva, L., Setiawan, J. 2007, A\&A, 473, 979

Pinsonneault, M. H., DePoy, D. L., \& Coffee, M. 2001, ApJ, 556, L59

Pollack, J. B., Hubickyj, O., Bodenheimer, P., Lissauer, J. J., Podolak, M., Greenzweig, Y. 1996, Icarus, 124, 62

Ramírez, I., \& Meléndez, J. 2004, ApJ, 609, 417

Reddy, B. E., Lambert, D. L., Laws, C., Gonzalez, G., \& Covey, K. 2002, MNRAS, 335, 100

Santos, N. C., Israelian, G., \& Mayor, M. 2000, A\&A, 363, 228

Santos, N. C., Israelian, G., Mayor, M., Bento, J. P., Almeida, P. C., Sousa, S. G., \& Ecuvillon, A. 2005, A\&A, 437, 1127

Schuler, S. C., Margheim, S. J., Thirupathi, S., Asplund, M., Smith, V. V., Cunha, K., \& Beers, T. C. 2008, AJ, 136, 2244

Smith, V. V., Lambert, D. L., \& Nissen, P. E. 1998, ApJ, 506, 405

Sneden, C. 1973, Ph.D. thesis, University of Texas, Austin

Stein, R. F., \& Nordlund, A. 1998, ApJ, 499, 914

Thévenin, F. 1990, A\&AS, 82, 179T

Udry, S., \& Santos, N. C. 2007, ARA\&A, 45, 397

Van Hamme, W. 1993, AJ, 106, 2096 